\title{
The Design and Implementation of a Network Simulated Virtual Laboratory Based on Dynamips
}

\author{
http://dx.doi.org/10.3991/ijoe.v12i02.5043
}

\author{
JIANG Guocui
}

Anhui Vocational college of Electronics \& Information Technology, Anhui, China

\begin{abstract}
This project designed a virtual experiment system for a computer network curriculum with Dynamips. First, this paper introduces the Dynampis function and running mode. Then it presents the system module design. Finally, this paper implements the Dynamips with Flex and Applet. System testing shows the virtual experiment system satisfies the network simulation function as the system was designed. The remote courseware virtual experiment system had great value in the teaching and learning process of the computer network curriculum.
\end{abstract}

Index Terms-Network Simulated, Virtual Laboratory, Dynamips

\section{INTRODUCTION}

Experimenting is a major step in scientific research, and also an essential part in teaching, especially in colleges and universities. Experiments are a crucial method for students to develop their knowledge. With the rapid development of technology in the Internet and in computer science, remote instruction or distance teaching has gradually taken on a bigger and bigger part in the students' learning process due to great flexibility in time and place [1-4]. The system described in this paper can create a platform on the Internet for students to experiment with computer network equipment and also provides a platform for the students and teacher to communicate online. The system is mainly achieved with Dynamips technology.

Dynamips originally was called Cisco 7200 Simulator, and the reason for creating Dynamips is simulating the Cisco 7200 router in a traditional X86 series [5]. Now the Dynamips simulators are able to simulate the Cisco C3600 series (including 3620364, 3620364), C3700 series (including 3725374), C2600 series (including 2610 to 2650 $\mathrm{xm}, 2610$ ), and $\mathrm{C} 1700$ series (containing 1710 and 1760) router platforms through the exchange of modules in some type of router simulator. The Dynamips also can simulate switches.

Dynamips mainly have the following three functions: (1) The simulator can be used as a training platform to help students become familiar with Cisco network equipment with little cost. This training will reach the goals of using real equipment. (2) The simulator can be used for experimenting or testing Cisco IOS's powerful features. (3) The simulator can test or verify a real network configuration and implementation in advance.

Dyanmips can simulate a Cisco router after compiling its runs in two modes: the first is host based and the other is set up with a virtual machine management port through hypervisor. The host-based mode directly loads runtime parameters to simulate a specific virtual router. The advantage of this mode is it runs without any external software; the disadvantage is the parameters of the virtual machine cannot be changed after startup. The hypervisor mode can create, start up, and shut down the virtual machine though the management port. The advantage of this mode is it can manage multiple virtual machines together. The disadvantages are when one of the virtual machine collapses, it will endanger the whole platform to collapse and the supervisor mode also needs to install Python to run Dynamips. This project chose the host-based mode because of its high equipment usage efficiency.

\section{SYSTEM MODULES DESIGN}

\section{A. System modules}

According to the system design goal, the function of the computer network virtual laboratory system mainly covers three areas: user authentication and management, course content learning and management modules, and virtual experiment simulation module, shown in Figure 1.

The user authentication and management module mainly function for certification and to distinguish the system user permissions. It includes user registration, user login and logout, review of the users' identity, editing users' information, and communication between uses i.e., text messages between households, etc.

The course content learning and management module mainly functions for planning the curriculum resources in a reasonable manner and includes the experiment content, class courseware, announcements, and BBS content as well as reviews the simulation projects, publishes simulation projects, and registers experimental equipment.

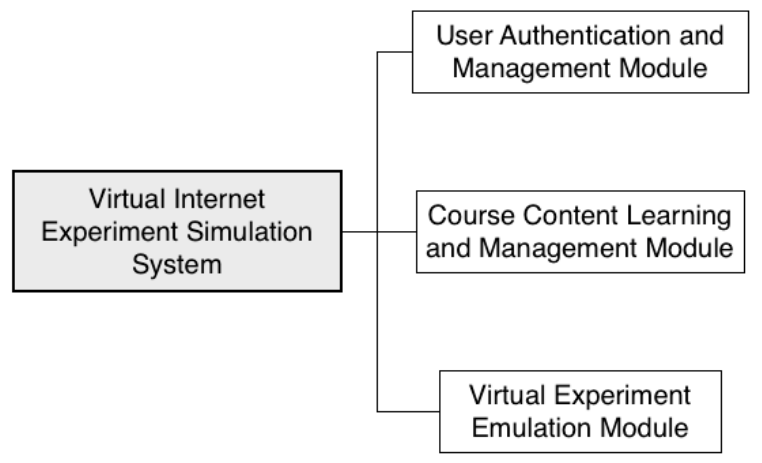

Figure 1. System modules 


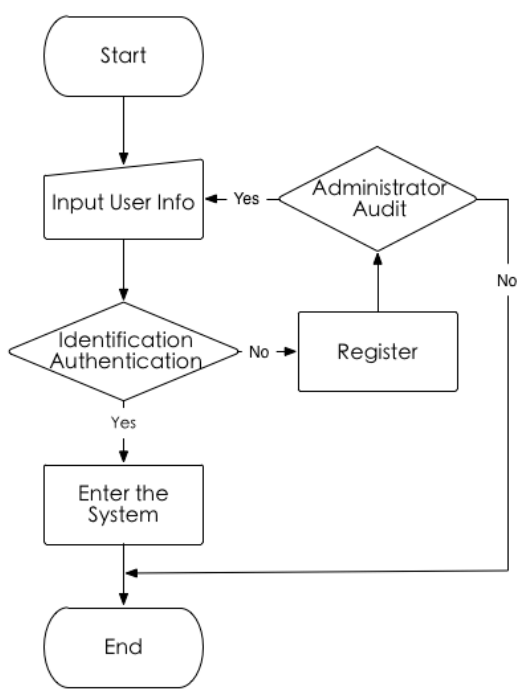

Figure 2. The user authentication flow chart.

The virtual experiment simulation module provides a virtual hardware environment. The user can operate on the topology of the virtual laboratory equipment, including drag, delete, and add equipment in the topology; set up a topology connection to the experiment equipment; and publish it.

\section{B. The user authentication and management module}

For a computer network virtual experiment system, users can be divided into three categories: students, teachers, and guests. Teachers and administrators in this system function similarly, so there would not be a subdivision. The system only allows registered users to log in, as unregistered users can only browse without access to the virtual reality function. The user authentication flow chart is shown in Figure 2.

\section{Course content learning and management module}

According to user role analysis, all users have access to the curriculum content, curriculum resources, and curriculum changes. Students and administrators can undertake online discussion. Only the administrators can review the curriculum statics, manage curriculum content and resources, and publish simulation projects. The module structure of the course content learning and management module is shown in Figure 3.

\section{Virtual experiment simulation module}

Based on the three basic characteristics of virtual reality: immersion, interactivity and constructive, the virtual simulation model has similar requirements. Students can be completely immersed in the virtual environment, the experimental data and results are close to the real experimental environment, the virtual environment will inspire understanding of the experiment content for the students such as the experiment of the route device. First, students can edit in the diagram, drag the composition to form a network, and then click on the equipment to enter the con sole interface, which is a simulation of the real console interface. Finally students can operate the console to get experiment feedback and results with no differences from a real environment.

In this project, the virtual simulation server used Dynamips to generate the virtual routers and switches. The

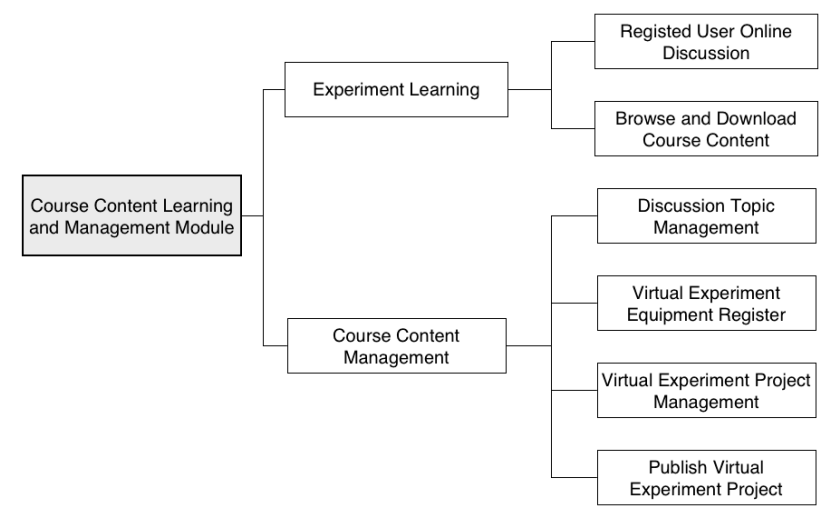

Figure 3. The structure of the course content learning and management module

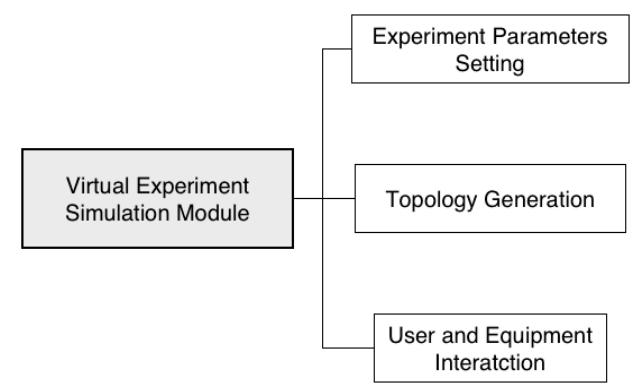

Figure 4. Structure of the virtual experiment simulation module

process of entering the virtual simulation module is as follows. (1) A user in the browser connects to the Web server to generate the topology of various parameters. (2) According to the parameters given by the user, the Web server generates an editable network topology. (3) The user clicks on the equipment on the topology network to finish the connection through the server.

The virtual experiment simulation module consists of three functions: experimental parameter configuration, topology generation, and user and equipment interaction, as shown in Figure 4.

\section{SYSTEM IMPLEMENTATION}

\section{A. Dynamips topology implementation based on Flex}

The topology rendering module, according to the requirement, has two functions: the first is the display function. It displays the topology structure in the browser according to the equipment connection information. The second is the control function, which provides the ability to edit and delete equipment in the topology for remote connection. The module is implemented through Flex 3.0 language in Flex Builder 3.0.

The topology display must have a topology data model first. The data model should contain two basic elements in topology: nodes and connections. This project used a modified XML format to represent the topology data model, for example a simplified topology network composed of five routers, the 5.XML content is

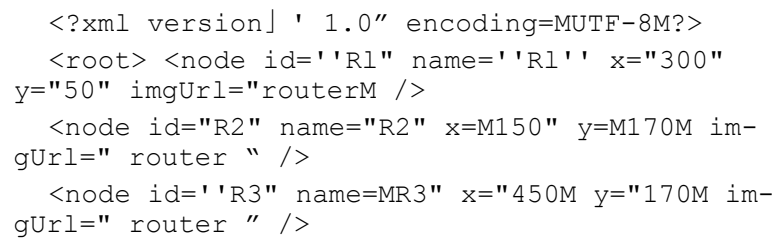




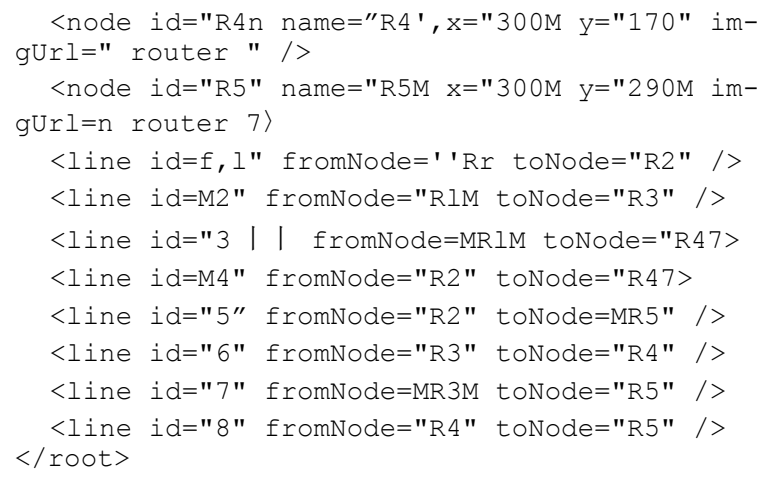

The topology of the XML file is shown in Figure 5.

\section{B. Dynamips remote connection implementation based on Applet}

The implementation of the remote connection to the Dynamips virtual machine is based on an Applet Telnet client. There are two main function modules: the NVT command execution work function and the Socket connection function. For the NVT command execution function, this project used a Java class Telnet Protocol Handler for its implementation. The Socket connection was completed in the Applet class life cycle.

Because Applet programs are downloaded from the website, they pose a great security concern. If the user enables the Java program in the browser, the Applet program will automatically download and execute without a chance to confirm or terminate. Due to the powerful function of Applet, the chance of a malicious program in the Applet program cannot be ruled out. This project used a sandbox to restrict the Applet program. The environment of the JAVA application and the Applet program are shown in Figure 6.

\section{System TESTING}

Users can right-click the equipment on the topology to call a Telnet client based on the Applet. The client can configure the Dynamips equipment through the graphical interface to realize the experiment requirements. According to RIP (Routing Information Protocol) to conduct the testing, the results show the route in addition to its three direct connected networks. It also uses RIP to go through the topology network and runs the command "show IP route" on the route to check the routing table information. The routing table information is consistent with the expected information. It shows the practical value of the virtual experiment system.

\section{CONCLUSION}

This paper analyzed the network simulated virtual machine system design and requirements. The computer network virtual experiment system was based on a Dynamips design and implemented in this project. Compared to other virtual machines, the simulation is closer to a real network. The system based on $\mathrm{B} / \mathrm{S}$ architecture and implemented with RIP technology improves the users' experience. The system solves equipment problems, finds shortages in the computer network curriculum, and solves the problem of poor simulation effects in a network virtual machine.

This system still has a lot of shortcomings. The system does not use an existing application framework, such as the well-known CakePHP and ThinkPHP; therefore, the expansibility of this system is not very good. The system uses a lot of Web technology and will require a heavy workload for maintenance. Improving the system's expansibility and maintainability will be a focus in future work. Also, the remote connection provided by Dynamips uses plaintext with a Telnet service; therefore, a more secure SSH connection should be adopted.

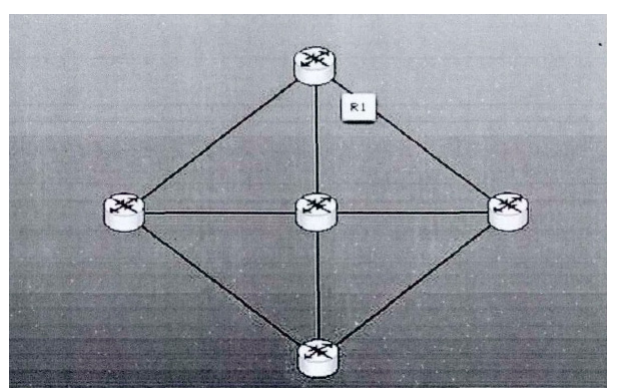

Figure 5. The topology of the 5.XML file

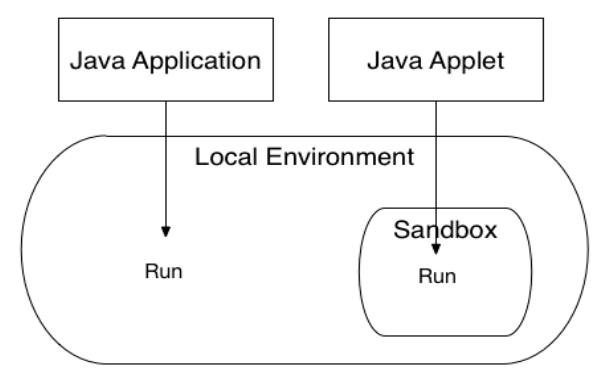

Figure 6. The sandbox structure of the JAVA and Applet application

\section{REFERENCES}

[1] Abazari, F., \& Analoui, M. (2014). Exploring the effects of virtual machine placement on the transmission of infections in cloud. InTelecommunications (IST), 2014 7th International Symposium on (pp. 278-282). IEEE. http://dx.doi.org/10.1109/ISTEL.2014. $\underline{7000714}$

[2] Autefage, V., \& Magoni, D. (2012). Network emulator: a network virtualization testbed for overlay experimentations. In Computer Aided Modeling and Design of Communication Links and Networks (CAMAD), 2012 IEEE 17th International Workshop on (pp. 266-270). IEEE. http://dx.doi.org/10.1109/CAMAD.2012. 6335347

[3] Chidambaram, B., \& Yang, Y. (2012). Cyber Analysis System Toolkit: A high-fidelity, virtual cyber test-bed for network modeling and experimentation. In MILITARY COMMUNICATIONS CONFERENCE, 2012-MILCOM 2012 (pp. 1-5). IEEE. http://dx.doi.org/10.1109/MILCOM.2012. 6415831

[4] Gamess, E., Urbáez, J., \& Palacios, A. (2011). A Visual Teaching and Learning Environment for the Open Shortest Path First Routing Protocol. In Proceedings of the Ninth Latin American and Caribbean Conference (LACCEI'2011). Medellin, Colombia.

[5] Hardy, J., Liu, L., Antonopoulos, N., Liu, W., Cui, L., \& Li, J. (2012). Assessment and evaluation of internet-based virtual computing infrastructure. InObject/Component/Service-Oriented RealTime Distributed Computing (ISORC), 2012 IEEE 15th International Symposium on (pp. 39-46). IEEE. http://dx.doi.org/10.1109/ISORC.2012.14

\section{AUTHOR}

JIANG Guocui is with the Anhui Vocational College of Electronics \& Information Technology, Anhui, China.

Submitted 17 September 2015. Published as resubmitted by the author 23 January 2016. 